\title{
An optimal adaptive finite element method
}

\author{
Rob Stevenson \\ Korteweg-de Vries Institute for Mathematics \\ Faculty of Science \\ University of Amsterdam
}




\section{Contents}

- model problem + (A)FEM

- newest vertex bisection

- convergence of AFEM

- quasi-optimal computational complexity 


\section{Optimal adaptive finite element methods}

Model problem: Poisson, 2D, newest vertex bisection.

[Generalizations: $\nabla \cdot \mathbf{A} \nabla$ with $\mathbf{A}$ symm. pos. def. piecewise constant, any space dimension $n$, red-refinements.]

Given $f \in H^{-1}(\Omega)$, find $u \in H_{0}^{1}(\Omega)$,

$$
a(u, v):=\int_{\Omega} \nabla u \cdot \nabla v=\int_{\Omega} f v=: f(v) \quad\left(v \in H_{0}^{1}(\Omega)\right) .
$$

$\|\cdot\|:=a(\cdot, \cdot)^{\frac{1}{2}}$.

$\tau$ conforming part. of $\Omega$ into triangles, $V_{\tau}=H_{0}^{1}(\Omega) \cap \prod_{T \in \tau} P_{d-1}(T)$. Find $u_{\tau} \in V_{\tau}$,

$$
a\left(u_{\tau}, v_{\tau}\right)=f\left(v_{\tau}\right) \quad\left(v_{\tau} \in V_{\tau}\right) .
$$

AFEM: GALSOLVE, compute a post. error est, MARK, REFINE. 


\section{Newest vertex bisection}

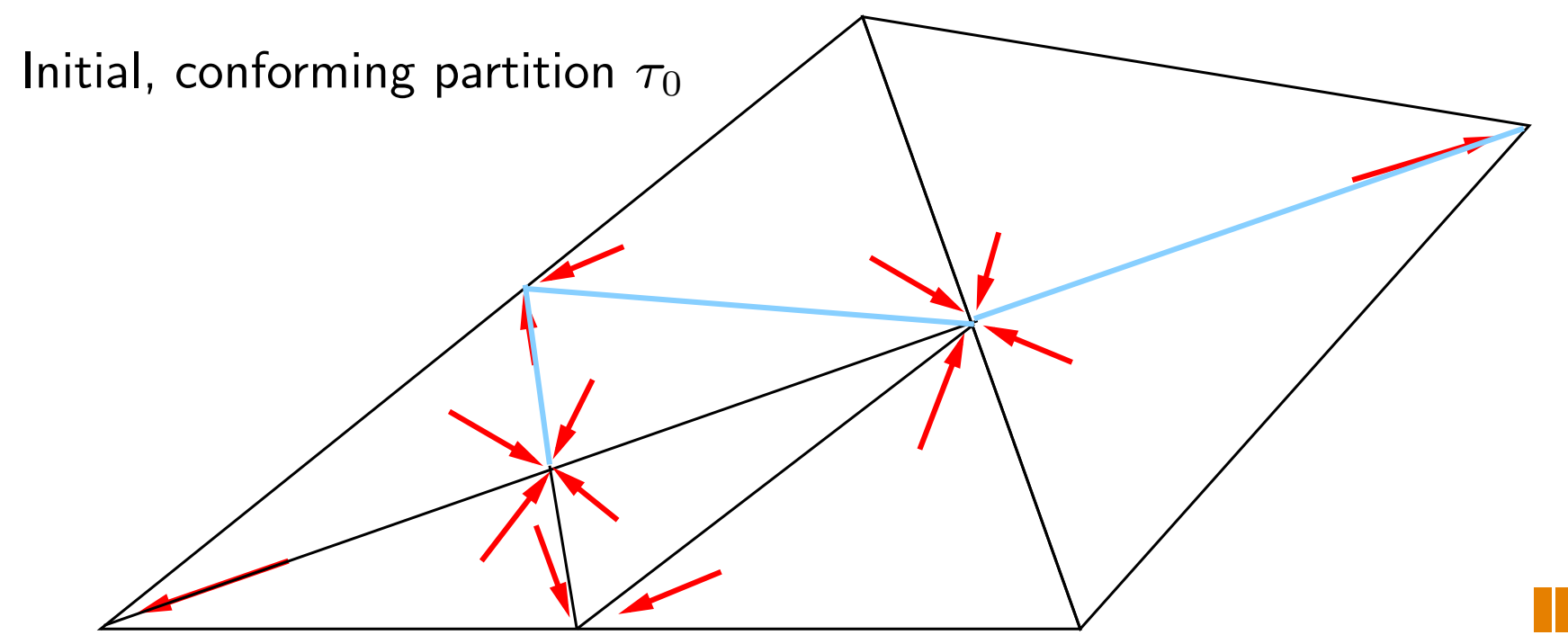

$\tau:=\operatorname{REFINE}[\tau, M]:$ Bisect all $T \in M \subset \tau$ a few times. Complete.

Th 1 (Binev, Dahmen, DeVore '04). With suitable assignment of newest vertices in initial mesh,

$$
\left.\# \tau_{K}-\# \tau_{0} \lesssim \sum_{i=0}^{K-1} \# M_{i} \quad \text { (unif. in } K\right) .
$$

(can be generalized to any space dimension [St.'08]) 


\section{Perspective: Approximation classes}

$\mathcal{A}_{\infty}^{s}:=\left\{u \in H_{0}^{1}(\Omega):|u|_{\mathcal{A}_{\infty}^{s}}:=\sup _{N} N^{s} \inf _{\left\{\tau \in \mathcal{P}: \# \tau-\# \tau_{0} \leq N\right\}}\left\|u-u_{\tau}\right\|_{H^{1}}<\infty\right\}$,

[i.e. $\left\|u-u_{\tau}\right\|_{H^{1}} \leq \varepsilon$ generally requires $\# \tau-\# \tau_{0} \leq \varepsilon^{-1 / s}|u|_{\mathcal{A}_{\infty}^{s}}^{1 / s}$. ] $\mathbf{I}$

When $\mathcal{P} \sim$ unif. refs, then

$$
u \in \mathcal{A}_{\infty}^{(d-1) / n} \Longleftarrow u \in H^{d}(\Omega) \text {, i.e., } \partial^{\alpha} u \in L_{2}(\Omega), \forall|\alpha| \leq d
$$

With $\mathcal{P} \sim$ all partitions created by newest vertex bisection:

$$
\begin{array}{r}
u \in \mathcal{A}_{\infty}^{(d-1) / n} \Longleftarrow u \in B_{q}^{d}\left(L_{p}(\Omega)\right), \text { any } p>\left(\frac{d-1}{n}+\frac{1}{2}\right)^{-1} \text { i.e., } \partial^{\alpha} u \in L_{p}(\Omega) \\
\forall|\alpha| \leq d
\end{array}
$$

([Binev, Dahmen, DeVore, Petrushev '02]) I

Regul. th: For suff sm $f, n=2, u$ in such a Besov space for any $d$ ([Dahlke, DeVore '97]). 


\section{A posteriori error estimator}

$\eta_{T}\left(f, u_{\tau}\right):=\operatorname{diam}(T)^{2}\left\|f+\triangle u_{\tau}\right\|_{L_{2}(T)}^{2}+\operatorname{diam}(T)\left\|\llbracket \nabla u_{\tau} \cdot \mathbf{n} \rrbracket_{\partial T}\right\|_{L_{2}(\partial T)}^{2}$,

Th 2 (Babuška, Rheinboldt '78; Verfürth '96).

$$
\left\|u-u_{\tau}\right\| \leq C_{1} \mathcal{E}\left(\tau, f, u_{\tau}\right):=C_{1}\left[\sum_{T \in \tau} \eta_{T}\left(f, u_{\tau}\right)\right]^{\frac{1}{2}} .
$$

Proof. $\left\|u-u_{\tau}\right\|=\sup _{v \in H_{0}^{1}(\Omega)} \frac{a\left(u-u_{\tau}, v\right)}{\|v\| \|}$.

$$
\begin{aligned}
& a\left(u-u_{\tau}, v\right)=a\left(u-u_{\tau}, v-v_{\tau}\right)=\int_{\Omega} f\left(v-v_{\tau}\right)-a\left(u_{\tau}, v-v_{\tau}\right) \\
& =\sum_{T \in \tau} \int_{T}\left(f+\triangle u_{\tau}\right)\left(v-v_{\tau}\right)-\int_{\partial T} \nabla u_{\tau} \cdot \mathbf{n}\left(v-v_{\tau}\right) .
\end{aligned}
$$

Th 3 (St.'06, Cascon,Kreuzer, Nochetto,Siebert '07).

$\sigma \supset \tau, R_{\tau \rightarrow \sigma}:=\{T \in \tau: T \notin \sigma\}$,

$$
\left\|u_{\sigma}-u_{\tau} \mid\right\| \leq C_{1}\left[\sum_{T \in R_{\tau \rightarrow \sigma}} \eta_{T}\left(f, u_{\tau}\right)\right]^{\frac{1}{2}} .
$$

Note $\# R_{\tau \rightarrow \sigma} \lesssim \# \sigma-\# \tau$. 
We call $\sigma \supset \tau$ a full refinement with respect to $T \in \tau$, when

$T$ and all its neighbours in $\tau$, as well as all faces of $T$ contain a vertex of $\sigma$ in their interiors.

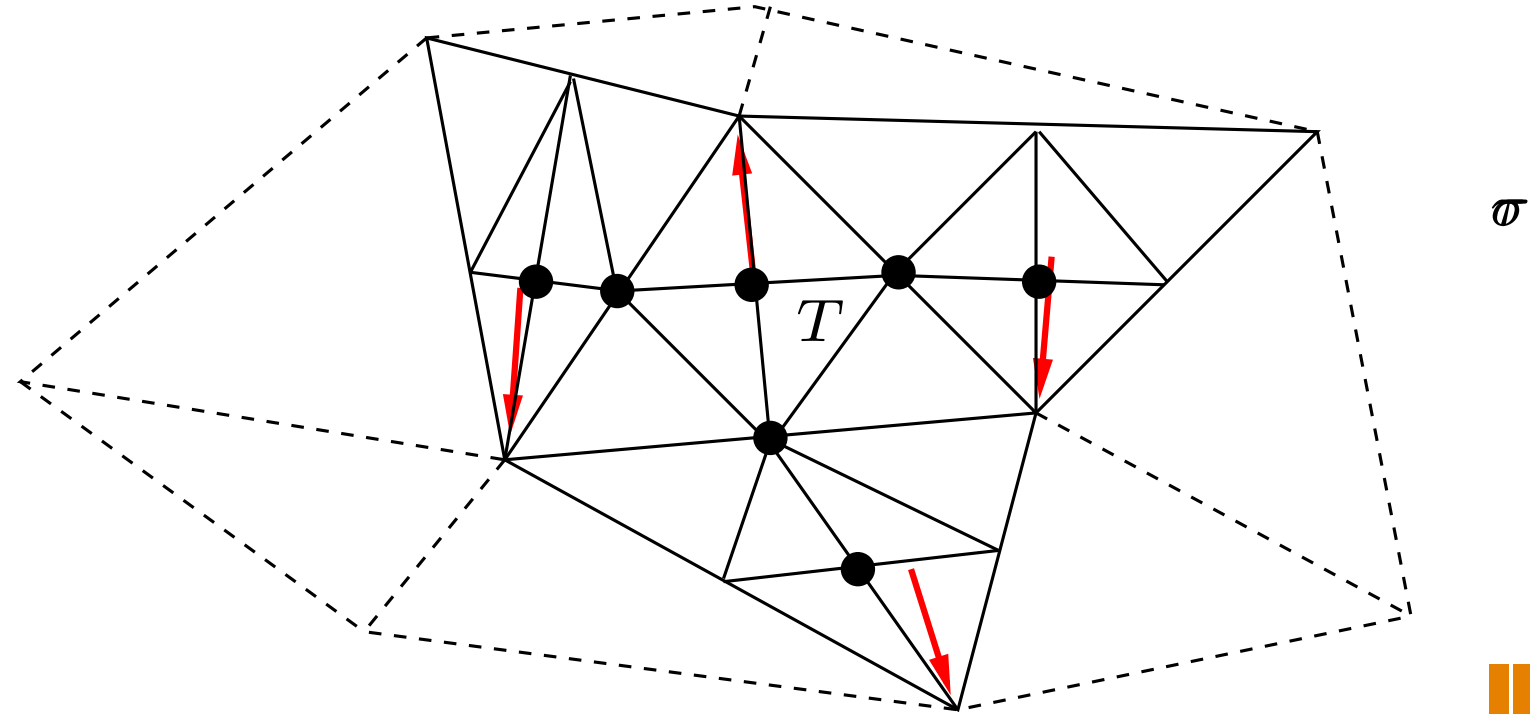

Th 4 (Morin, Nochetto, Siebert '00). Let $f \in \prod_{T \in \tau} P_{d-2}(T), \sigma \supset \tau$ full ref w.r.t. $T \in \tau$. Then

$$
\eta_{T}\left(f, u_{\tau}\right) \lesssim \sum_{\tilde{T} \in\{T\} \cup\{\text { neighbours }\}}\left|u_{\sigma}-u_{\tau}\right|_{H^{1}(\tilde{T})}^{2}
$$


Corol 5. $\sigma \supset \tau$ full ref w.r.t. $T \in M \subset \tau$. Then

$$
c_{2}\left[\sum_{T \in M} \eta_{T}\left(f, u_{\tau}\right)\right]^{\frac{1}{2}} \leq\left\|u_{\sigma}-u_{\tau}\right\| \| \quad\left(\text { not true for any } f \in L_{2}(\Omega)\right)
$$

In part, $c_{2} \mathcal{E}\left(\tau, f, u_{\tau}\right) \leq\left\|u-u_{\tau}\right\|$.

AFEM converges (Dörfler '96, Morin, Nochetto, Siebert '00)

$\operatorname{MARK}\left[\tau, f, u_{\tau}\right] \rightarrow M:$ Let $\theta \in(0,1]$. Select smallest $M \subset \tau$ s.t.

$$
\left[\sum_{T \in M} \eta_{T}\left(\tau, f, u_{\tau}\right)\right]^{\frac{1}{2}} \geq \theta \mathcal{E}\left(\tau, f, u_{\tau}\right) \text {. }
$$

$\operatorname{REFINE}[\tau, M] \rightarrow \sigma:$ Construct smallest (conforming) $\sigma \supset \tau$ that is a full refinement w.r.t. all $T \in M$.

$$
\begin{gathered}
\|\| u-u_{\tau}\left\|^{2}=\right\| \mid u-u_{\sigma}\left\|^{2}+\right\| u_{\sigma}-u_{\tau} \|^{2} \\
\left\|u_{\sigma}-u_{\tau}\right\| \mid \geq c_{2} \theta \mathcal{E}\left(\tau, f, u_{\tau}\right) \geq \frac{c_{2} \theta}{C_{1}}\left\|u-u_{\tau}\right\| \| \\
\leadsto\left|\left\|u-u_{\sigma}\right\|\right| \leq\left(1-\frac{c_{2}^{2} \theta^{2}}{C_{1}^{2}}\right)^{\frac{1}{2}}\left\|u-u_{\tau}\right\|
\end{gathered}
$$

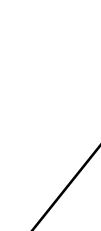




\section{AFEM converges with optimal rate}

[St '06] ([Binev, Dahmen,DeVore '04] using coarsening)

$\operatorname{AFEM}[f, \varepsilon] \rightarrow\left[\tau_{m}, u_{\tau_{m}}\right] \quad \%$ let $f \in \prod_{T \in \tau_{0}} P_{d-2}(T)$

compute $\mathrm{Gal}$ sol $u_{\tau_{0}} \in V_{\tau_{0}} ; k:=0$

while $C_{1} \mathcal{E}\left(\tau_{k}, f, u_{\tau_{k}}\right)>\varepsilon$ do

$M_{k}:=\operatorname{MARK}\left[\tau_{k}, f, u_{\tau_{k}}\right]$

$\tau_{k+1}:=\operatorname{REFINE}\left[\tau_{k}, M_{k}\right]$

compute Gal sol $u_{\tau_{k+1}} \in V_{\tau_{k+1}}$

$k:=k+1$

end do

$m:=k$

$$
\sim\left\|u-u_{\tau_{m}} \mid\right\| \leq \varepsilon, \quad \# \tau_{m}-\# \tau_{0} \lesssim \sum_{k=0}^{m-1} \# M_{k}
$$

Th 6. If $\theta<\frac{c_{2}}{C_{1}}$, then

$$
\begin{aligned}
\# M_{k} & \leq \inf \left\{\# \rho-\# \tau_{0}:|| u-u_{\rho}\left\|\left|\leq\left[1-\frac{C_{1}^{2} \theta^{2}}{c_{2}^{2}}\right]^{\frac{1}{2}}\left\|\left|u-u_{\tau_{k}} \|\right|\right\}\right.\right.\right. \\
& \leq\left[\left[1-\frac{C_{1}^{2} \theta^{2}}{c_{2}^{2}}\right]^{\frac{1}{2}}|| u-u_{\tau_{k}} \|\right]^{-1 / s}|u|_{\mathcal{A}_{\infty}^{s}}^{1 / s} \text { when } u \in \mathcal{A}_{\infty}^{s} . \\
\sim \# \tau_{m}-\# \tau_{0} & \lesssim \sum_{k=0}^{m-1}\left|\left\|u-\left.u_{\tau_{k}}||^{-1 / s}|u|_{\mathcal{A}_{\infty}^{s}}^{1 / s} \lesssim\left|\| u-u_{\tau_{m-1}}\right|\right|^{-1 / s}|u|_{\mathcal{A}_{\infty}^{s}}^{1 / s} \lesssim \varepsilon_{\varepsilon_{8 / 16}}^{-1 / s}|u|_{\mathcal{A}_{\infty}^{s}}^{1 / s} .\right.\right.
\end{aligned}
$$


Pr. Th. 6. Let $\sigma \supset \tau_{k}$ s.t. $\left\|u-u_{\sigma}\right\| \leq\left[1-\frac{C_{1}^{2} \theta^{2}}{c_{2}^{2}}\right]^{\frac{1}{2}} \mid\left\|u-u_{\tau_{k}}\right\|$. Th.3 shows

$$
\begin{aligned}
C_{1}^{2} \sum_{T \in R_{\tau_{k} \rightarrow \sigma}} \eta_{T}\left(f, u_{\tau_{k}}\right) & \geq\left\|u_{\sigma}-u_{\tau_{k}}\right\|^{2}=\left\|u-u_{\tau_{k}} \mid\right\|^{2}-\left\|u-u_{\sigma}\right\|^{2} \\
& \geq \frac{C_{1}^{2} \theta^{2}}{c_{2}^{2}}\left\|u-u_{\tau_{k}}\right\|^{2} \geq C_{1}^{2} \theta^{2} \mathcal{E}\left(\tau_{k}, f, u_{\tau_{k}}\right)^{2},
\end{aligned}
$$

i.e., $\left[\sum_{T \in R_{\tau_{k} \rightarrow \sigma}} \eta_{T}\left(f, u_{\tau_{k}}\right)\right]^{\frac{1}{2}} \geq \theta \mathcal{E}\left(\tau_{k}, f, u_{\tau_{k}}\right)$.I

$M:=\operatorname{MARK}\left[\tau_{k}, f, u_{\tau_{k}}\right]$ is smallest set with this prop, so

$$
\# M \leq \# R_{\tau_{k} \rightarrow \sigma} \leq \# \sigma-\# \tau_{k} .
$$

For arb. $\rho$ with $\left\|\left|u-u_{\rho}\left\|\leq\left[1-\frac{C_{1}^{2} \theta^{2}}{c_{2}^{2}}\right]^{\frac{1}{2}}\right\|\right| u-u_{\tau_{k}}\right\|$, take $\sigma:=\tau_{k} \cup \rho$. Then

$$
\# M \leq \# \sigma-\# \tau_{k} \leq \# \rho-\# \tau_{0} .
$$




\section{General right hand sides and inexact solves}

$\mathbf{R H S}[\tau, f, \delta] \rightarrow\left[\sigma, f_{\sigma}\right]$

$\%$ In: $\tau$ a partition, $f \in H^{-1}(\Omega)$ and $\delta>0$.

$\%$ Out: $f_{\sigma} \in \prod_{T \in \tau} P_{d-2}(T)$, where $\sigma=\tau$, or, if necessary, $\sigma \supsetneq \tau$, $\%$ such that $\left\|f-f_{\sigma}\right\|_{H^{-1}(\Omega)} \leq \delta$.

If $u \in \mathcal{A}_{\infty}^{s}$, cost of RHS will not dominate if $\exists c_{f}$ s.t. $\# \sigma-\# \tau \leq c_{f}^{1 / s} \delta^{-1 / s}$, and cost $\lesssim \# \sigma$. Such a pair $(f, \mathbf{R H S})$ is called $s$-optimal. For suff. smooth $f, s$-optimality with $s=\frac{d}{n}\left(>\frac{d-1}{n}\right)$ can be realized.

$\operatorname{GALSOLVE}\left[\tau, f_{\tau}, u_{\tau}^{(0)}, \delta\right] \rightarrow w_{\tau}$ $\%$ In: $\tau, f_{\tau} \in\left(\mathcal{S}_{\tau}\right)^{\prime}$, and $u_{\tau}^{(0)} \in \mathcal{S}_{\tau}$. $\%$ Out: $w_{\tau} \in \mathcal{S}_{\tau}$ with $\left\|u_{\tau}-w_{\tau}\right\|_{H^{1}(\Omega)} \leq \delta$.

$\%$ The call should require $\lesssim \max \left\{1, \log \left(\delta^{-1}\left\|u_{\tau}-u_{\tau}^{(0)}\right\|_{H^{1}(\Omega)}\right)\right\} \# \tau$ ops. 


\section{AFEM has opt compl}

$\operatorname{AFEM}[f, \varepsilon] \rightarrow\left[\tau_{m}, w_{\tau_{m}}\right]$

$\% \omega, \beta>0$ suff small constants.

$w_{\tau_{0}}:=0 ; k:=0 ; \delta_{0} \approx\|f\|_{H^{-1}(\Omega)}$

do

do $\delta_{k}:=\delta_{k} / 2$

$\left[\tau_{k}, f_{\tau_{k}}\right]:=\mathbf{R H S}\left[\tau_{k}, f, \delta_{k} / 2\right]$

$w_{\tau_{k}}:=$ GALSOLVE $\left[\tau_{k}, f_{\tau_{k}}, w_{\tau_{k}}, \delta_{k} / 2\right]$

if $\eta_{k}:=\left(2+C_{1} c_{2}^{-1}\right) \delta_{k} / 2+C_{1} \mathcal{E}\left(\tau_{k}, f_{\tau_{k}}, w_{\tau_{k}}\right) \leq \varepsilon$ then stop endif

until $\delta_{k} \leq \omega \mathcal{E}\left(\tau_{k}, f_{\tau_{k}}, w_{\tau_{k}}\right)$.

$M_{k}:=\operatorname{MARK}\left[\tau_{k}, f_{\tau_{k}}, w_{\tau_{k}}\right]$

$\tau_{k+1}:=\operatorname{REFINE}\left[\tau_{k}, M_{k}\right]$

$w_{\tau_{k+1}}:=w_{\tau_{k}}, \delta_{k+1}:=2 \beta \eta_{k}, k:=k+1$

enddo

$m:=k$

Th 7. $\left\|u-w_{\tau_{m}}\right\|_{H^{1}(\Omega)} \leq \varepsilon$. If $u \in \mathcal{A}_{\infty}^{s}$, and $(f$, RHS) is s-optimal, then both $\# \tau_{m}$ and work $\lesssim \varepsilon^{-1 / s}\left(|u|_{\mathcal{A}_{\infty}^{s}}^{1 / s}+c_{f}^{1 / s}\right)$. 


\section{Realizations of RHS}

ex. $f \in L_{2}(\Omega)$. $Q_{\sigma}$ being $L_{2}$-orth.proj. onto $\prod_{T \in \sigma} P_{d-2}(T)$.

$$
\left\|f-Q_{\sigma} f\right\|_{H^{-1}(\Omega)} \lesssim \sqrt{\sum_{T \in \sigma} \operatorname{vol}(T)^{\frac{2}{n}}\left\|f-Q_{T} f\right\|_{L_{2}(T)}^{2}}=: \operatorname{osc}(f, \sigma)
$$

Given $\bar{\theta} \in(0,1)$, run MARK and REFINE algorithm on osc until $\leq \delta$. Is quasi-optimal in the sense that whenever

$$
f \in \overline{\mathcal{A}}^{s}:=\left\{f \in L_{2}(\Omega): \sup _{N} N^{s} \inf _{\left\{\tau: \# \tau-\# \tau_{0} \leq N\right\}} \operatorname{osc}(f, \tau)<\infty\right\},
$$

then $(f, \mathbf{R H S})$ is $s$-optimal (assuming $\int_{T} f P_{d-2}$ exact in $\mathcal{O}(1)$ operations) So greedy works (thanks to factor $\operatorname{vol}(T)^{\frac{2}{n}}$ ). 
ex. General $d, n>1$. $\left(\frac{1}{2}+\frac{1}{n}\right)^{-1}<q \leq 2, f \in L_{q}(\Omega)\left(\hookrightarrow H^{-1}(\Omega)\right)$.

Generalization of previous case with

$$
\operatorname{osc}(f, \sigma):=\sqrt{\sum_{T \in \sigma} \operatorname{vol}(T)^{1-\frac{2}{q}+\frac{2}{n}} \inf _{p \in P_{d-2}(T)}\|f-p\|_{L_{q}(T)}^{2}} .
$$

ex. $n=2, d=2, f(v)=\int_{K} v, K$ a smooth curve. $\operatorname{RHS}[\tau, f, \delta] \rightarrow\left[\sigma, f_{\sigma}\right]$ : Refine those $T$ that intersect $K$ until their diameters $\lesssim \delta^{2} .\left.\left(f_{\sigma}\right)\right|_{T}:=$ $\frac{\text { length }(K \cap T)}{\operatorname{vol}(T)}$. $s$-optimal for $s=\frac{1}{2}\left(=\frac{d-1}{n}\right)$. 


\section{References}

[1] P. Binev, W. Dahmen, and R. DeVore. Adaptive finite element methods with convergence rates. Numer. Math., 97(2):219 - 268, 2004.

[2] P. Binev, W. Dahmen, R. DeVore, and P. Petruchev. Approximation classes for adaptive methods. Serdica Math. J., 28:391-416, 2002.

[3] S. Dahlke and R. DeVore. Besov regularity for elliptic boundary value problems. Comm. Partial Differential Equations, 22(1 \& 2):1-16, 1997.

[4] W. Dörfler. A convergent adaptive algorithm for Poisson's equation. SIAM J. Numer. Anal., 33:1106-1124, 1996.

[5] P. Morin, R. Nochetto, and K. Siebert. Data oscillation and convergence of adaptive FEM. SIAM J. Numer. Anal., 38(2):466-488, 2000. 
[6] R.S. Optimality of a standard adaptive finite element method. Found. Comput. Math., 7(2):245-269, 2007.

[7] R.S. The completion of locally refined simplicial partitions created by bisection. Math. Comp., 77:227-241, 2008.

[8] R. Verfürth. A Review of A Posteriori Error Estimation and Adaptive Mesh-Refinement Techniques. Wiley-Teubner, Chichester, 1996. 


\section{Extensions}

- [Cascon,Kreuzer,Nochetto,Siebert '07]: One bisection of marked cells suffices (no interior node). Marking for reducing osc can be omitted, assuming $f \in L_{2}(\Omega)$, exact integration, and with exact solving of Gal systems.

- L. Chen, M.J. Holst, and J. Xu. Convergence and optimality of adaptive mixed finite element methods. Accepted by Mathematics of Computation, 2007.

- Y. Kondratyuk, R. S. An optimal adaptive algorithm for the Stokes problem. To appear in SIAM J. Numer. Anal. 